\title{
Actitudes hacia el consumo de alcohol y consumo de alcohol en estudiantes de enfermería'
}

\author{
Juan Yovani Telumbre-Terrero² \\ Santiaga Enriqueta Esparza-Almanza³ \\ Bertha Alicia Alonso-Castillo ${ }^{4}$ \\ María Teresa de Jesús Alonso-Castillo ${ }^{5}$
}

doi:10.11144/Javeriana.ie19-2.ahca

Cómo citar: Telumbre-Terrero JY, Esparza-Almanza SE, Alonso-Castillo BA, AlonsoCastillo MTJ. Actitudes hacia el consumo de alcohol y consumo de alcohol en estudiantes de enfermeria. Investig Enferm Imagen Desarr. 2017;19(2):69-81. http://dx.doi. org/10.11144/Javeriana.ie19-2.ahca

1. Artículo original de investigación. Fecha de recepción: 11 de noviembre de 2015. Fecha de aceptación: 16 de mayo de 2016.

2. Maestro en Ciencias de Enfermeria. Profesor y coordinador del Servicio Social de la Licenciatura en Enfermería de la Facultad de Ciencias de la Salud de la Universidad Autónoma del Carmen, México. Correo electrónico: jtelumbre@pampano.unacar.mx

3. Maestra en Ciencias de Enfermería. Profesora y subdirectora del Pregrado de la Facultad de Enfermería de la Universidad Autónoma de Nuevo León (UANL), México. Correo electrónico: sesparza54@yahoo.com.mx

4. Doctora en Bioética. Profesora de la Facultad de Enfermería de la Universidad Autónoma de Nuevo León, México. Correo electrónico: balonso_mx@yahoo.com.mx

5. Doctora en Bioética. Profesora y colaboradora del Cuerpo Académico de Prevención de Adicciones de la Facultad de Enfermería de la Universidad Autónoma de Nuevo León, México. Correo electrónico: talonso_55@hotmail.com 


\section{Resumen}

Objetivo: Determinar la relación de las actitudes hacia el consumo de alcohol y el consumo de alcohol en estudiantes de enfermería. Método: Estudio descriptivo correlacional. El muestreo fue censal con una muestra de 166 participantes de un programa de la Licenciatura en Enfermería de dos universidades privadas de Monterrey, Nuevo León, México. Se utilizó la Escala de Actitudes hacia el Consumo de Alcohol y el Cuestionario de Identificación de Trastornos debidos al Consumo de Alcohol. Resultados: Se identificó que el $83,1 \%$ de los estudiantes de enfermería han consumido alcohol alguna vez en la vida, el 73,5\% lo ha hecho en el último año y el 33,7\% señaló haber consumido en los últimos siete días. Las actitudes positivas hacia el consumo de alcohol se relacionaron positiva y significativamente con el consumo sensato $\left(r_{\mathrm{s}}=0,401 ; p=0,001\right)$, con el consumo dependiente $\left(r_{\mathrm{s}}=0,263 ; p=0,001\right)$ y con el consumo dañino de alcohol $\left(r_{\mathrm{s}}=0,219 ; p=0,016\right)$. Conclusión: Los hallazgos muestran la necesidad de diseñar y desarrollar intervenciones de enfermería para prevenir el consumo de alcohol y otras drogas y fomentar el desarrollo de habilidades para resistir el consumo de alcohol.

Palabras clave: consumo de bebidas alcohólicas; estudiante de enfermeria; actitud

\section{Attitudes toward Alcohol Consumption and Alcohol Consumption among Nursing Students}

\section{Abstract}

Objective: To determine the relationship of attitudes towards alcohol and alcohol consumption among nursing students. Method: correlational descriptive study. The sampling was census with a sample of 166 participants of a program of Nursing of two private universities in Monterrey, Nuevo Leon, Mexico. The techniques used to gather data were the Scale for the Measurement of Attitudes Towards Alcohol and the alcohol Use Disorders Identification Test. Results: It was identified that $83.1 \%$ of nursing students have consumed alcohol sometime in life, $73.5 \%$ have done so in the last year and $33.7 \%$ reported having used in the last seven days. Positive attitudes towards alcohol consumption were associated positively and significantly with the sensible consumption $\left(r_{\mathrm{s}}=0.401 ; p=0.001\right)$, with dependent consumption $\left(r_{\mathrm{s}}=0.263 ; p=0.001\right)$ and the harmful use of alcohol $\left(r_{\mathrm{s}}=0.219 ; p=0.016\right)$. Conclusion: The findings show the need to design and develop nursing interventions to prevent alcohol and other drugs consumption and encourage the development of skills to avoid alcohol.

Keywords: alcoholic beverages; nursing student; attitude 


\section{Atitudes frente ao uso de álcool e uso de álcool entre estudantes de enfermagem}

\section{Resumo}

Objetivo: Determinar a relação das atitudes frente ao uso de álcool e uso de álcool em estudantes de enfermagem. Método: Estudo descriptivo correlacional. A amostragem foi por recenseamento com amostra de 166 participantes do programa da Licenciatura em Enfermagem de duas universidades privadas de Monterrey, Nuevo León, México. A Escala de Atitudes frente ao Uso de Álcool foi utilizada e o Teste de Identificação de Transtornos devidos ao Uso de Álcool. Resultados: Identificou-se que 83,1\% dos estudantes de enfermagem já usaram álcool alguma vez na vida, 73,5\% no último ano e $33,7 \%$ relatou ter usado nos últimos sete dias. As atitudes positivas frente ao uso de álcool relacionaram-se positiva e significativamente com uso sensato $\left(r_{\mathrm{s}}=0,401\right.$; $p=0,001)$, com uso dependente $\left(r_{\mathrm{s}}=0,263 ; p=0,001\right)$ e com uso nocivo de álcool $\left(r_{\mathrm{s}}=\right.$ $0,219 ; p=0,016)$. Conclusão: Os achados revelam a necessidade de criar e desenvolver intervenções de enfermagem para prevenir o uso de álcool e outras drogas e encorajar o desenvolvimento de habilidades para resistir o uso de álcool.

Palauras-chave: uso de bebidas alcoólicas; estudante de enfermagem; atitude 


\section{Introducción}

El consumo de alcohol constituye un creciente problema de salud pública, debido a su alto grado de adicción, aunado a que es la sustancia psicoactiva más utilizada por la población en general, la cual provoca múltiples consecuencias biológicas, psicológicas y sociales en el individuo, las familias y la sociedad (1). La Organización Mundial de la Salud (2) señala que el consumo de drogas es uno de los factores de riesgo para la salud global, donde el consumo de alcohol representa un factor significativo en la tasa general de morbilidad.

En México, cerca de 27 millones de personas han ingerido alcohol en grandes cantidades por ocasión de consumo, con frecuencias que oscilan entre una vez al mes y a diario (1). La Encuesta Nacional de Adicciones 2011 (ENA) reporta que el consumo de alcohol alguna vez en la vida en población general de 12 a 65 años es del 71,3\%, que el consumo de alcohol en el último año fue del 51,3\% y que en el último mes fue del 31,6\%. Además, el 32,8\% de la población presenta un consumo alto de alcohol; el 0,8\%, un consumo diario; el 5,4\%, consumo consuetudinario, y el 6,2\%, dependencia (3).

El presente estudio centra su atención en los estudiantes de enfermería, quienes - además de encontrarse en la etapa de la juventud- serán futuros profesionales de la salud y están inmersos en el contexto cultural donde se presenta consumo de alcohol, tabaco y otras drogas (4). Una proporción considerable de estudiantes de enfermeria, aun cuando no ha concluido su proceso académico formativo, se encuentra incorporada al mercado laboral y es probable que enfrente situaciones como desgaste físico y emocional, excesiva carga horaria, mayor responsabilidad laboral, privación de la convivencia familiar y diversión (5). Además, durante el proceso de cuidar se enfrenta a dilemas éticos derivados del cuidado, el sufrimiento humano y los procesos de muerte, factores que pueden aumentar la probabilidad de afrontar estas realidades por medio del consumo de alcohol (6).

En este sentido, diversos estudios realizados en Brasil, Honduras y Colombia muestran que entre el 30\% y el 98\% de los estudiantes de enfermería han consumido alcohol alguna vez en la vida, con un tipo de consumo de bajo riesgo en la mayoría de los estudiantes y mayor consumo en los hombres que en las mujeres (7-9). La frecuencia de consumo es de dos veces por semana, con hasta diez bebidas alcohólicas en una sola ocasión $(8,9)$.

Desde la perspectiva de enfermería, el Modelo de Promoción de la Salud (MPS) de Nola J. Pender lo han utilizado ampliamente los profesionales de enfermeria, porque permite entender los comportamientos de las personas relacionados con la salud y el bienestar, el incremento de la competencia protectora opuesta al riesgo y al daño ocasionado por conductas no saludables como el consumo de drogas. Además, este modelo orienta hacia la comprensión de los factores implicados en la toma de decisión de las personas para mantener su salud y evitar las conductas de riesgo, como el consumo de alcohol.

E1 MPS expone que la actitud es un aspecto que interviene en la realización o modificación de la conducta en los seres humanos (10). Afirma que la conducta es racional y que las actitudes son un componente decisivo 
que representan el análisis de los comportamientos voluntarios dirigidos para conseguir un logro. Cuando existen actitudes claras, concretas y definidas por realizar una conducta, aumenta la probabilidad de lograrla. El MPS hace hincapié en el nexo entre características personales, experiencias previas, cogniciones y afectos, aspectos situacionales y circunstanciales que se vinculan con los comportamientos; estas últimas son determinantes a la hora de decidir o asumir un comportamiento saludable o de riesgo para la salud, debido a su alto nivel de interiorización y la manera de ver la realidad que lo rodea (11).

Las actitudes de los estudiantes de enfermería hacia el consumo de alcohol las han abordado diversos estudios realizados en Brasil $(12,13)$, donde se ha reportado que los estudiantes presentan actitudes positivas hacia el consumo de esta sustancia que, a la vez, incrementa su consumo en los jóvenes. Señalan que les ayuda a pasar momentos agradables, relaja las tensiones del día a día y produce placer (14).

Considerando que los estudiantes de enfermería se encuentran expuestos a una mayor información sobre el cuidado a la salud y las acciones para evitar el consumo de drogas, es importante conocer las actitudes hacia el consumo de alcohol. Lo anterior es de utilidad para identificar áreas de formación futura en los planes de estudio de enfermería, enfocadas en teorías de enfermería que contribuyan a analizar los factores que motivan o influyen en las conductas saludables y no saludables como el MPS. Así mismo, se espera proponer intervenciones de enfermería dirigidas a la promoción de la salud y a la prevención del consumo de alcohol. Los resultados de este estudio permitirán sustentar intervenciones específicas a futuro, dirigidas a la prevención del consumo de drogas y la promoción de la salud. Con base en lo anterior, el propósito del presente estudio es describir la relación de las actitudes hacia el consumo de alcohol y el consumo de alcohol en estudiantes de enfermería.

\section{Método}

El diseño del estudio fue descriptivo-correlacional (15). La población estuvo constituida por 172 estudiantes de un programa de licenciatura en enfermería de dos universidades privadas del área Metropolitana de Monterrey, Nuevo León. El tipo de muestreo fue censal, es decir, se contó con la participación de todos los estudiantes de ambas escuelas. El tamaño de muestra para el estudio fue de $n=166$, debido a que seis personas no se encontraron en el momento de la recolección de datos. Para ello se utilizó una ficha de datos personales y de consumo de alcohol, en la cual se recabaron datos de identificación, características sociodemográficas e información sobre el consumo de alcohol.

Así mismo, se empleó el Cuestionario de Identificación de Trastornos debidos al Consumo de Alcohol (AUDIT), el cual es una prueba que se aplica para examinar el uso de alcohol durante los últimos doce meses y sus consecuencias. Está constituido por diez reactivos: los reactivos del uno al tres determinan la cantidad y frecuencia de consumo de alcohol; los del cuatro al seis exploran la posibilidad de que exista dependencia al alcohol; y los del 
siete al diez exploran el consumo dañino de alcohol (16). La escala es de cero a cuarenta puntos. A mayor puntaje, existe mayor consumo de alcohol. Si se obtiene un valor de uno a tres, se considera un consumo sin riesgo (consumo sensato); si se obtienen de cuatro a siete puntos, se tiene problemas con el consumo de alcohol (consumo dependiente); y si se registran de ocho a cuarenta puntos, se considera un consumo con riesgo elevado o consumo dañino. El AUDIT es un instrumento con validez transcultural que ha sido probado en población mexicana con una sensibilidad del 90\%, una especificidad del $94 \%$ y una confiabilidad aceptable (17). Este cuestionario obtuvo un alfa de Cronbach de 0,77 en este estudio.

Para la evaluación de las actitudes se utilizó el cuestionario diseñado por García y López-Sánchez (18) que, por medio de trece preguntas, aborda diversas concepciones (positivas y negativas) hacia el consumo de alcohol. La escala de respuesta es tipo Likert, que van de muy de acuerdo a totalmente en desacuerdo. Los reactivos $5,6,7,10,11$ y 13 corresponden a actitudes positivas, y los reactivos $1,2,3,4,8,9$ y 12, a actitudes negativas hacia el consumo de alcohol. Se asignó el valor de 5 puntos para la respuesta "muy de acuerdo", 4 puntos para la opción "de acuerdo", 3 puntos para la opción "indiferente", 2 puntos para la respuesta "en desacuerdo" y 1 punto para la respuesta "muy en desacuerdo" para las actitudes negativas, y para las actitudes positivas se invierte los valores.

La puntuación final de las actitudes incluye la sumatoria de cada reactivo, donde se puede obtener un valor mínimo de 13 y máximo de 65. Para su interpretación, puntajes entre 13 y 39 se consideran actitudes positivas hacia el consumo de alcohol, y entre 40 y 65 , actitudes negativas hacia el consumo de alcohol. Los autores reportan un test-retest de $r=0,638$ ( $p<0,001)$. La versión original de la escala se encuentra en castellano y portugués, y se ha utilizado en estudiantes de secundaria y bachillerato de España y Portugal. En este estudio, la escala de obtuvo una consistencia interna de 0,81.

El estudio contó con la aprobación de las comisiones de Ética e Investigación de la Facultad de Enfermería de la Universidad Autónoma de Nuevo León. Así mismo, el presente estudio se apegó a lo dispuesto en el Reglamento de la Ley General de Salud en Materia de Investigación para la Salud en seres Humanos en México. Se consideró el título segundo de los aspectos éticos de la investigación en seres humanos, capítulo I, artículos 13, 14, 16, 17, 20, 21, 36, 57 y 58 (19).

Posteriormente, se solicitó autorización por escrito a las instituciones educativas; una vez otorgados los permisos, se procuraron las listas de los grupos. Una vez identificados dichos grupos, se visitó a los estudiantes para invitarlos a participar en el estudio, explicándoles de forma clara y sencilla los objetivos de la investigación.

A los estudiantes que decidieron participar, y previo a la aplicación de los instrumentos, se les entregó un consentimiento y asentimiento informado, para asegurar la autorización de los padres o tutores y de los participantes en el estudio. Se orientó a los estudiantes sobre la importancia de 74 sus respuestas; por ello, de forma respetuosa, se les pidió que contestaran lo más cercano a su realidad. Además, se les reiteró que su participación 
era totalmente confidencial y anónima. Al finalizar el llenado de los instrumentos, se les pidió los insertaran en un sobre y que lo depositaran en el contenedor que estaba al frente al aula. Finalmente, se agradeció a los participantes su tiempo y la información.

La captura y análisis de los datos se llevó a cabo en el paquete estadístico Statistical Package of Social Sciences (SPSS) versión 20. Se utilizó estadística descriptiva e inferencial no paramétrica en virtud de los resultados de la prueba de normalidad en la distribución de los datos.

\section{Resultados}

En relación con las características sociodemográficas de los participantes, se identificó que el 68,7\% corresponde al género femenino, el 91\% está soltero y el $62 \%$ presenta una edad entre 17 y 21 años. Por grado escolar, el 40,4\% corresponde al primer año, y el 36,7\%, al segundo.

Respecto al consumo de alcohol de los estudiantes, se observó una media de edad de inicio del consumo a los 16,8 años (DE $=2,9)$. Además, los participantes refirieron consumir en promedio 5,3 ( $\mathrm{DE}=4,6)$ bebidas alcohólicas en una sola ocasión. En la tabla 1 se observa que el 83,1\% (IC 95\%: $0,77-0,89)$ de los estudiantes de enfermería han consumido alcohol alguna vez en la vida, y el 73,5\% (IC 95\%: 0,67-0,80) lo ha hecho en el último año. Cabe destacar que el 33,7\% señaló haber consumido en los últimos siete días.

TABLA 1. Prevalencia de consumo de alcohol $(\mathrm{n}=166)$

\begin{tabular}{|l|r|r|r|r|r|r|}
\hline \multirow{2}{*}{ Prevalencia de consumo } & \multicolumn{2}{|c|}{ Si } & \multicolumn{2}{c|}{ No } & \multicolumn{2}{c|}{ IC 95\% } \\
\cline { 2 - 7 } & \multicolumn{1}{|c|}{ f } & \multicolumn{1}{c|}{ \% } & \multicolumn{1}{c|}{ f } & \multicolumn{1}{c|}{ \% } & \multicolumn{1}{c|}{ LI } & \multicolumn{1}{c|}{ LS } \\
\hline Alguna vez en la vida & 138 & 83,1 & 28 & 16,9 & 77 & 89 \\
\hline En el último año & 122 & 73,5 & 44 & 26,5 & 67 & 80 \\
\hline En el último mes & 88 & 53,0 & 78 & 47,0 & 45 & 61 \\
\hline En los últimos siete días & 56 & 33,7 & 110 & 66,3 & 26 & 41 \\
\hline
\end{tabular}

f: frecuencia; IC: intervalo de confianza; LI: limite inferior; LS: limite superior.

Fuente: elaboración propia.

Por lo que corresponde al tipo de consumo de alcohol en los estudiantes de enfermeria, en la tabla 2 se muestra que predomina el consumo sensato $(36,7 \%)$, seguido del consumo dependiente $(32,8 \%)$.

TABla 2. Tipo de consumo de alcohol según el AUDIT ( $\mathrm{n}=122)$

\begin{tabular}{|l|r|r|}
\hline \multicolumn{1}{|c|}{ Tipo de consumo } & f & \multicolumn{2}{|c|}{$\%$} \\
\hline Sensato & 45 & 36,9 \\
\hline Dependiente & 40 & 32,8 \\
\hline Dañino & 37 & 30,3 \\
\hline
\end{tabular}

f: frecuencia.

Fuente: elaboración propia. 
Las actitudes hacia el consumo de alcohol presentaron una media de $36,1(\mathrm{DE}=5,8)$. Por tipo de actitudes predominaron las negativas, con una media de 22,5 ( $\mathrm{DE}=5,3)$, sobre las positivas hacia el consumo de alcohol, con una media de 13,55 (DE $=5,5)$.

El $12 \%$ de los participantes está muy de acuerdo y el 13,3\% está de acuerdo en beber alcohol con los amigos y amigas, siempre que pueda. El $10,2 \%$ de los participantes está muy de acuerdo y el 21,7\% de acuerdo en comprar alcohol para su uso y el de sus amigos. Por otro lado, el 24,1\% está muy de acuerdo y el 33,1\% de acuerdo en convencer a los demás sobre los peligros de las bebidas alcohólicas. El 21,1\% de los participantes está muy de acuerdo y el $29,5 \%$ de acuerdo en participar en cualquier campaña contra las bebidas alcohólicas. E1 50,6\% de los estudiantes está muy de acuerdo y el $16,9 \%$ está de acuerdo en impedir que un niño beba alcohol.

Se encontró que las actitudes positivas hacia el consumo de alcohol se relacionan positiva y significativamente con el consumo de alcohol de acuerdo con el AUDIT $\left(r_{s}=0,393 ; p<0,01\right)$, con el consumo sensato $\left(r_{s}=0,401\right.$; $p<0,01)$, el dependiente $\left(r_{s}=0,263 ; p<0,03\right)$ y el dañino $\left(r_{s}=0,219 ; p<0,16\right)$.

\section{Discusión}

Se identificó que los participantes inician el consumo de alcohol, en promedio, a los 16 años de edad, lo cual concuerda con Braga y Bastos (6), quienes indican que la población inicia a consumir alcohol a los 17 años o menos. Esto se debe, tal vez, a que los jóvenes se encuentran en un periodo de transición entre la adolescencia y la adultez, en el cual el joven se siente miembro y partícipe de una "cultura de edad", caracterizada por sus propios comportamientos, valores, normas, espacios y modas. Por ello es probable que busque la conducta del consumo de alcohol como una forma de iniciar el proceso de adultez (20).

Los estudiantes de enfermería consumen, en promedio, cinco bebidas alcohólicas, lo cual es similar a los resultados de López (7) y Matute y Pillon (8). Esta situación es preocupante, porque los participantes son estudiantes de enfermería de quienes se espera sean modelos de rol y educadores en salud, ya que se encuentran en una posición única para impulsar estrategias de prevención y abandono del consumo de drogas, aparte de que conocen los factores de riesgo que implica para su salud y la de las personas a quienes brindan el cuidado (21).

El consumo de alcohol alguna vez en la vida y en último año fue menor en comparación con los hallazgos de Montoya (22), que reporta prevalencias del $98 \%$ y del 90\%, respectivamente. El consumo de alcohol en el último mes y en los últimos siete días fue mayor en relación con los hallazgos de Bautista y colaboradores (23), quienes reportan prevalencias inferiores al $15 \%$. Lo anterior pudiera estar relacionado con la interacción de variables sociodemográficas, ambientales y culturales propias del contexto universitario que facilitan y promueven el consumo excesivo de alcohol (22).

Referente al tipo de consumo de alcohol, los estudiantes de enfermería presentaron un consumo sensato en un $36,9 \%$, un consumo dependiente del $32,8 \%$ y un consumo dañino del $30,2 \%$. Los resultados coinciden con los 
de López y colaboradores (7) y Martínez y colaboradores (24), quienes reportan una mayor proporción de alumnos con consumo sensato y el consumo dependiente y dañino en menores proporciones en estudiantes de enfermería de Brasil y Colombia. Esto se deba quizás a que socialmente la conducta de consumo de alcohol en grandes cantidades está relacionada con los jóvenes, especialmente los fines de semana, como una forma de socializar (25).

Las actitudes negativas hacia el consumo de alcohol fueron mayores que las actitudes positivas. Estos resultados difieren de los De Vargas $(12,13)$, quien reporta que los estudiantes de enfermería visualizan el consumo de alcohol como algo normal, agradable y que relaja las tensiones del día a día. En relación con las actitudes negativas hacia el consumo de alcohol, los jóvenes consideran que estarían dispuestos a convencer a los demás sobre los peligros de las bebidas alcohólicas, a colaborar en campañas contra las bebidas alcohólicas, a impedir que los niños consuman alcohol y a que el consumo de alcohol se prohíba. Las actitudes positivas que sobresalen es que los estudiantes estarían dispuestos a beber alcohol con los amigos y amigas siempre que puedan, además de comprar alcohol para su uso y el de los amigos.

Lo anterior reafirma el hecho de que los jóvenes usan el alcohol para facilitar la interacción social, la expresividad verbal, la desinhibición de la conducta y como reductor de la tensión física y psicológica. Estos efectos brindan la posibilidad al joven de ser reconocido por el grupo de pares en el que se desenvuelve $(26,27)$. Esta disposición de los estudiantes de enfermería pudiera explicarse porque durante su formación adquieren los conocimientos sobre los efectos negativos del consumo de alcohol en el organismo, los cuales se van construyendo progresivamente (28). Además, el profesional enfermero es considerado un agente clave en programas de promoción y prevención de consumo de drogas (29).

Hay que considerar que el MPS señala la existencia de factores cognitivos perceptuales, entendidos como aquellas concepciones, creencias e ideas que tienen las personas sobre la salud que la llevan o inducen a conductas o comportamientos determinados, y que son modificados por las condiciones situacionales, personales e interpersonales de cada sujeto y el resultado de esta evaluación puede ser la adquisición de conductas favorecedoras o desfavorecedoras de la salud (10).

Se encontró una relación positiva de las actitudes (positivas) hacia el consumo de alcohol con los tipos de consumo de alcohol y con la cantidad de bebidas alcohólicas consumidas en un día típico. Estos hallazgos concuerdan con Guzmán y colaboradores (30) y Villegas y colaboradores (31) en jóvenes universitarios y de zonas marginales. Tal y como lo determina el modelo teórico, la actitud para el consumo de alcohol está determinada por el conjunto de creencias sobre las consecuencias que provoca el consumir alcohol y la evaluación que el individuo les atribuye. Esas creencias pueden reforzarse en los jóvenes que ya tienen una experimentación previa de consumo, lo que los llevará a tener una actitud más favorable, basada no solo en las creencias individuales, sino también en la experiencia de consumo, lo que puede potenciar la actitud positiva hacia el consumo $(10,11,30)$. 
Hay que resaltar que el MPS retoma las características y las experiencias individuales, además de la valoración de las creencias en salud, en razón a que estas últimas son determinantes a la hora de decidir un comportamiento saludable o de riesgo para la salud, debido a su alto nivel de interiorización y la manera de ver la realidad que lo rodea. La valoración de las creencias en salud relacionadas con los conocimientos y experiencias previas determinan las conductas adoptadas por la persona; estas creencias están dadas por los beneficios percibidos, las barreras para la acción, la autoeficacia y las emociones, motivaciones, deseos o propósitos $(10,11)$.

López y colaboradores (1) señalan que en adolescentes y jóvenes existe un mayor impacto de la actitud por el peso significativo cultural de la opinión de los amigos y compañeros de escuela para la toma de decisiones sobre la conducta de consumo de alcohol; así mismo, se evidencia que la actitud los jóvenes hacia el consumo de alcohol, tabaco u otras drogas será más favorable cuando menor sea la percepción del riesgo, mayores sean las creencias distorsionadas y más permisiva sea la disposición para su consumo.

\section{Conclusión}

Los resultados de la presente investigación aportan conocimientos sobre la relación de las actitudes y el consumo de alcohol en estudiantes de enfermería. Del total de los estudiantes de enfermería, el 73,5\% ha consumido alcohol alguna vez en la vida, y el 33,7\% en los últimos siete dias, en que predomina un consumo sensato de alcohol, seguido del dependiente y el dañino. El consumo de alcohol se inicia, en promedio, a los 16,6 años y los estudiantes consumen, en promedio, 5,3 bebidas alcohólicas en un día típico. Se destaca que predominan las actitudes negativas hacia el consumo de alcohol. Así mismo, las actitudes positivas hacia el alcohol se relacionan positivamente con su consumo.

Con base en los hallazgos donde los estudiantes presentaron un tipo de consumo sensato de alcohol, se recomienda implementar programas educativos para evitar la transición a otro tipo de consumo. Así mismo, diseñar y desarrollar intervenciones de enfermería para prevenir el consumo de alcohol y otras drogas, a través del fortalecimiento de las actitudes negativas hacia el consumo de alcohol y fomentar el desarrollo de habilidades para resistir el consumo de alcohol; de igual manera, es necesario continuar investigando la relación entre estas variables con el empleo de diferentes metodologías y en otros grupos poblacionales.

\section{Financiamiento}

Ninguno.

\section{Conflictos de interés}

Los autores declaran no tener conflictos de interés. 


\section{Referencias}

1. López-Cisneros MA, Villar LMA, Alonso CMM, Alonso CMTJ, Rodríguez AL. Attitudes towards consumption and non-consumption of alcohol among high school students in México. Rev Esc Enferm USP. 2013;47(4):815-21.

2. Organización Mundial de la Salud. Alcohol [internet]. 2011 [citado 9 abr 2016]. Disponible en: http://www.who.int/mediacentre/factsheets/ fs349/es/

3. Instituto Nacional de Psiquiatría Ramón de la Fuente Muñiz, Instituto Nacional de Salud Pública, Secretaría de Salud. Encuesta Nacional de Adicciones 2011: reporte de alcohol. [internet]. 2012. Disponible en: http://www.conadic.salud.gob.mx/pdfs/ENA_2011_ALCOHOL.pdf

4. Pinos PN, Inocenti MA, Renato TC. Consumo de benzodiacepinas sin prescripción médica en los/as estudiantes de primer año de la escuela de enfermería de la Universidad de Guayaquil, Ecuador. Rev LatinoAm Enfermagem. 2008;16(número especial):634-9.

5. Barbosa DE, Ferreira FAR. El trabajo del estudiante de enfermería como un factor de riesgo para el consumo de alcohol y otras drogas. Rev Latino-Am Enfermagem. 2008;16(número especial):565-71.

6. Braga AB, Bastos AF. Formação do acadêmico de enfermagem e seu contato com as drogas psicoativas. Texto \& Contexto Enfermagem [internet]. 2005;13(2):241-9. Disponible en: http://www.redalyc.org/ articulo.oa?id=71413207

7. López-Maldonado MC, Villar LMA, Gherardi-Donato ECD. Consumo de drogas lícitas en estudiantes de enfermería de una universidad privada en Bogotá, Colombia. Rev Latino-Am Enfermagem. 2011;19(número especial):707-13.

8. Matute RC, Pillon SC. Uso de bebidas alcohólicas entre estudiantes de enfermería en Honduras. Rev Latino-am Enfermagem. 2008;16(número especial):584-9.

9. Tam E, Benedita C. El consumo de alcohol y el estrés entre estudiantes del segundo año de enfermería. Rev Latino-Am Enfermagem. 2010;18(número especial):496-503.

10. Giraldo OA, Toro RMY, Macías LAM, Valencia GCA, Palacio RS. La promoción de la salud como estrategia para el fomento de estilos de vida saludables. Hacia Promoc Salud. 2010;15(1):128-43.

11. Aristizábal HGP, Blanco BDM, Sanchez RA, Ostiguín MRM. The model of health promotion proposed by Nola Pender. A reflection on your understanding. Enferm Univ. 2011;8(4):128-43.

12. De Vargas D. Attitudes of nursing students facing questions related to alcohol, alcoholism and the alcoholic. Acta Paul Enferm. 2011;24(5):638-44.

13. Vargas D, Bittencourt MN. Álcool e alcoolismo: atitudes de estudantes de enfermagem. Rev Brasileira Enfermagem. 2013;66(1):84-9.

14. García SBA. Actitud, norma subjetiva, y control conductual como predictores del consumo de drogas en jóvenes de zona marginal 2009 
[tesis de maestría]. Nuevo León: Facultad de Enfermería. Universidad Autónoma de Nuevo León México.

15. Burns N, Grove SK. Diseños de investigación. En: Investigación en enfermería. Madrid: Elsevier; 2003. p. 209-51.

16. Babor T, Higgin-Biddle J, Saunders J, Monteiro M. The alcohol use disorders identification test. En: Guidelines for use in primary care. 2nd ed. New York: World Health Organization; 2002.

17. De La Fuente J, Kershenovich D. El alcoholismo como problema médico. Rev Facultad de Medicina UNAM. 1992;35(2):47-51.

18. García RJA, López-Sánchez C. Estilos de vida y salud: promoción y prevención. En: Terol MC, Quiles Y, Pérez V, coordinadores. Manual de evaluación psicosocial en contextos de salud. Madrid: Pirámide; 2012. p. 161-173.

19. Secretaría de Salud. Reglamento de la Ley de Salud en Materia de Investigación para la salud [Internet]. México; 1987 [citado $10 \mathrm{abr}$ 2016]. Disponible en: http://www.salud.gob.mx/unidades/cdi/nom/ compit/rlgsmis.html

20. Musitu OG, Pons DJ. Adolescencia y alcohol: buscando significados en la persona, la familia y la sociedad. Madrid: Entimema; 2010.

21. Cid P, Pimenta AM. Percepción de los beneficios del consumo de drogas y barreras para su abandono entre estudiantes del área de salud. Rev Latino-Am Enfermagem. 2008;16 (número especial):621-6.

22. Montoya VEM, Cunningham J, Brans B, Strike C, Miotto, WMD. Consumo percibido y uso de drogas lícitas e ilícitas en estudiantes universitarios de Medellín, Colombia. Rev Latino-am Enfermagem. 2009;17:886-92.

23. Bautista PF, Simich L, Strike C, Brandns B, Giesbrecht N, Khenti A. Policonsumo simultáneo de drogas en estudiantes de pregrado del área de la salud de una Universidad de el Salvador. Texto \& Contexto Enfermagem. 2011;21(número especial):56-62.

24. Martínez RMR, Coronado HMJ, Betancourt EMC, Díaz OA, Gallegos MJ. Drogas lícitas e ilícitas: consumo de los estudiantes de la Facultad de Enfermería. Enferm Neurol. 2010;10(3):130-4.

25. Díaz MA, Díaz MLR, Hernández-Ávila CA, Narro RJ, Fernández VH, Solís TC. Prevalencia del consumo riesgoso y dañino de alcohol y factores de riesgo en estudiantes universitarios de primer ingreso. Salud Mental. 2008;31(4):271-82.

26. Armendáriz GNA, Villar LMA, Alonso CMM, Alonso CBA, Oliva RNN. Eventos estresantes y su relación con el consumo de alcohol en estudiantes universitarios. Investig Enferm Imagen Desarr. 2012;14(2):97-112.

27. Londoño PC, García HW, Valencia LSC, Vinaccia AS. Expectativas frente al consumo de alcohol en jóvenes universitarios colombianos. Anales de Psicología [internet]. 2005;21(2):259-67. Disponible en: http://www.um.es/analesps/v21/v21_2/07-21_2.pdf

28. Lastre AG, Gaviria GG, Arrieta R. Conocimiento sobre el consumo de sustancias psicoactivas en estudiantes de enfermería. Cienc Innov Salud. 2013;1(1):46-51. 
29. Vargas D, Villar LMA. Álcool, alcoolismo e alcoolista: concepções e atitudes de enfermeiros de unidades básicas distritais de saúde. Rev Latino-Am Enfermagem. 2008;16(número especial):543-50.

30. Guzmán FFR, García SBA, Rodríguez AL, Alonso CMM. Attitude, subjective norm, and behavioral control as predictors of drug use among youth in marginal urban area of northern México. Frontera Norte. 2013;26(51):53-74.

31. Villegas PMA, Alonso CMM, Benavides TR, Guzmán FFR. Consumo de alcohol y funciones ejecutivas en adolescentes: una revisión sistemática. Aquichan. 2013;13(2):234-46. 
